\title{
Development of Bifunctional Electrodes for Closed-loop Fuel Cell Applications
}

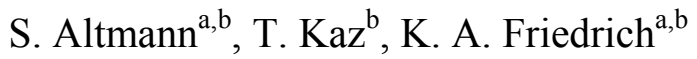 \\ a Institute of Thermodynamics and Thermal Engineering, University Stuttgart, \\ Pfaffenwaldring 6, 70550 Stuttgart, Germany \\ ${ }^{\mathrm{b}}$ Institute of Technical Thermodynamics, German Aerospace Center, \\ Pfaffenwaldring 38 - 40, 70569 Stuttgart, Germany
}

Unitized regenerative fuel cells (URFC) in combination with photovoltaic modules are attractive systems for space missions because of their extended operation times and low weight. Electrical energy, being stored throughout the planetary day, can be converted into electricity by the fuel cell during the night. An important topic for URFC development is the function of the socalled bifunctional oxygen electrodes which generally require a combination of favourable properties for fuel cell and electrolysis mode. In this work different oxygen electrode configuration for the operation in a unitized reversible fuel cell are compared. Different possibilities for bifunctional electrodes based on the primary catalysts are compared, namely a mixture of catalysts, layered structures with the two different catalyst layers, and segmented areas with single catalysts. It was shown that the mixture of both catalysts $\left(\mathrm{Pt}\right.$ and $\left.\mathrm{IrO}_{2}\right)$ performs best for the present stage of electrode development. Also multilayer electrodes yielded good results with a good potential for optimization.

\section{Introduction}

Fuel cells offer attractive benefits as energy supply devices for different portable and mobile applications. In comparison to batteries the energy density and the specific energy are higher and therefore extended operation times without external energy support can be realized. Also mass and volume benefits can be achieved in advanced stack and system designs. However, one drawback of fuel cells for portable applications is the need of an external continuous supply of hydrogen and oxygen. While the latter one can be taken out of air, the fuel needs an infrastructure with $\mathrm{H}_{2}$ production facilities and refilling stations.

By using a unitized regenerative fuel cell (URFC) a closed systems can be achieved. URFCs can regenerate fuel (hydrogen) and oxidant (oxygen) by water electrolysis with an external energy supply like a photovoltaic cell or a wind turbine. This combination can be considered as a zero emission energy supply and a closed loop system. In this respect, a URFC system is directly comparable to a secondary battery especially regarding user handling as well as regarding the charging and discharging behaviour.

While an ordinary hydrogen/air polymer electrolyte fuel cell simply uses Pt as catalyst for the hydrogen electrode, the electrode for the oxygen reactions is more complex. Pt shows superior reactivity in fuel cells but at the same time very high overpotentials in the electrolysis mode. In the literature (1-2) different alternative catalysts are proposed, primarily $\mathrm{Ir}, \mathrm{Ru}$, their oxides and combinations of them. Unfortunately the activity of these materials for oxygen reduction in the fuel cell mode is 
rather low. Hence the use of two catalysts is inevitable. In this work various electrode designs with $\mathrm{Pt}$ and $\mathrm{IrO}_{2}$ catalysts are being investigated.

\section{Approach}

The 3 different options, which are being compared in this study in both fuel cell (FC) and electrolysis mode (EC), are illustrated in Figure. 1.

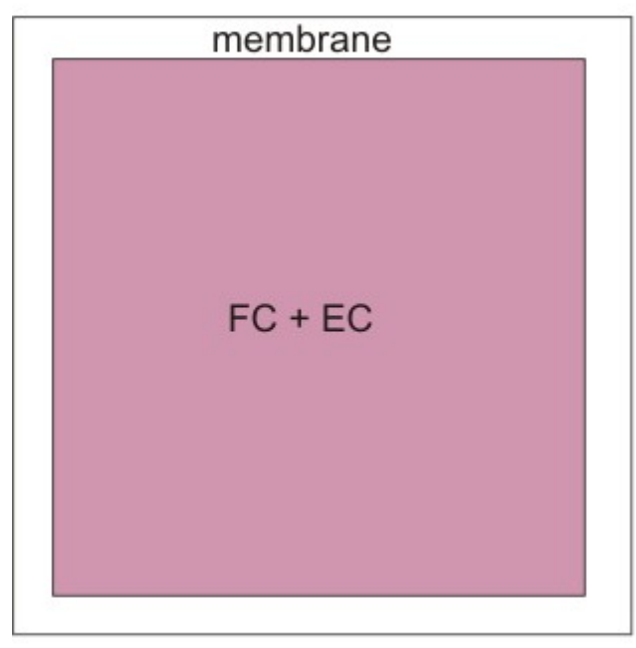

Figure 1. Schematic of different possibilities for bifunctional electrodes based on the primary catalysts. Left: Option 1 - Mixture of catalysts; Center: Option 2 - Layered structure with the two different catalyst layers; Right: Option 3 - Segmented areas with single catalysts

\section{Option 1 - Mixture}

A simple mixture of both materials is the most common configuration in literature (3-4). It is used as the reference design for this work.

The advantage of this option is the simple preparation of the MEA using only one production step. However, the mixture of two different catalysts for different chemical reactions has to involve compromises in structure and reactivity. In particular, since there are no specific reaction areas, the backing must be a compromise between hydrophobic (fuel cell mode) and hydrophilic (electrolysis mode) properties.

\section{Option 2 - Multilayered Electrode}

As an alternative to the mixture, two electrode layers, one for FC mode and for EC mode, are applied onto the membrane. During operation specific electrodes for both working directions are available. The order of the layers can be varied with either Pt in contact to the electrolyte (Option2_1), or in contact to the backing (Option2_2). In this multilayered electrode the electrical and the ionic resistances are very important issues. While the inner layer exhibits superior proton transfer to the membrane, the outer one has a better electronic conductivity to the bipolar-plate. To get a better understanding of the behaviour of the electrodes, the Option 2 MEAs were equipped with different loadings of 
$\mathrm{IrO}_{2}$. Following this approach it has to be considered that not only the number of catalyst particles but also the thickness of the catalyst layer changes.

The use of multilayer electrodes offers fully active areas of the electrode for each mode and thus good performance can be expected. On the other hand, the electrical and ionic connection between electrode, membrane and current collector can be a problem. Also the transport of gases or water could be reduced because of small pores in the outer layer. This is similar to the diffusion limitation at high current density known from generic fuel cells.

\section{Option 3-Segmented Electrode}

In the third design the active areas are split into separate zones for the fuel cell and the electrolysis reaction. Hence different configurations are possible, like few large areas (squared) or many smaller ones (stripes). In a further step the segment geometry can be adapted to the flow field.

The segmented electrode allows the use of partially optimized backings. Each part could have the right grade of hydrophobicity. In this configuration very pure catalysts are applied which yield very good performances. However, by dividing the electrode in different parts the active area is effectively reduced. Therefore the current and thus performance of the whole cell are expected to decrease.

\section{$\underline{\text { Experimental }}$}

All MEAs have been manufactured by the dry spraying technique developed at DLR (5). As catalysts Platinum (Pt) and Iridium-(IV)-oxide $\left(\mathrm{IrO}_{2}\right)$ are used. To increase the proton conductivity of the electrodes $30 \mathrm{wt} \%$ Nafion is added. The membrane of all MEAs is Nafion 1135, which have been pre-treated in $\mathrm{H}_{2} \mathrm{O}_{2}$ and $\mathrm{H}_{2} \mathrm{SO}_{4}$ before use. At the end of the preparation all MEAs have been hot rolled (temperature: $160{ }^{\circ} \mathrm{C}$, frequency: $0.6 \mathrm{~Hz}$ ).

Table I shows all MEAs used to compare the three options. All hydrogen electrodes with the exception of Option 1 have a low loading of $\mathrm{Pt}$, since it was not expected to influence the cell performance drastically.

TABLE I. Prepared MEAs

\begin{tabular}{|c|c|c|c|c|c|}
\hline \multirow{3}{*}{ Name } & \multicolumn{2}{|c|}{ Hydrogen } & \multicolumn{2}{|l|}{ Oxygen } & \multirow[t]{3}{*}{ Structure } \\
\hline & \multirow[t]{2}{*}{ Catalyst } & Loading & \multirow[t]{2}{*}{ Catalyst } & Loading & \\
\hline & & $\mathrm{mg} / \mathrm{cm}^{2}$ & & $\mathrm{mg} / \mathrm{cm}^{2}$ & \\
\hline Option 1 & Pt black & 1.56 & $\mathrm{IrO}_{2}+\mathrm{Pt}(1: 1)$ & 1.61 & Mixed \\
\hline Option 2_1 & Pt black & 0.70 & $\mathrm{Pt}$ and $\mathrm{IrO}_{2}$ & $0.93 / 0.78$ & Multilayer (Pt inside) \\
\hline Option 2_22 & Pt black & 0.70 & $\mathrm{IrO}_{2}$ and $\mathrm{Pt}$ & $0.78 / 0.87$ & Multilayer $\left(\mathrm{IrO}_{2}\right.$ inside $)$ \\
\hline Option 2_23 & Pt black & 0.67 & $\mathrm{IrO}_{2}$ and $\mathrm{Pt}$ & $0.62 / 1.00$ & Multilayer $\left(\mathrm{IrO}_{2}\right.$ inside $)$ \\
\hline Option 2_24 & Pt black & 0.67 & $\mathrm{IrO}_{2}$ and $\mathrm{Pt}$ & $1.07 / 1.00$ & Multilayer $\left(\mathrm{IrO}_{2}\right.$ inside) \\
\hline Option 2_25 & Pt black & 0.67 & $\mathrm{IrO}_{2}$ and $\mathrm{Pt}$ & $1.38 / 1.00$ & Multilayer $\left(\mathrm{IrO}_{2}\right.$ inside $)$ \\
\hline Option 3_11 & Pt black & 0.87 & $\mathrm{IrO}, / \mathrm{Pt}$ & $0.73 / 0.73$ & Seqmented (square) \\
\hline Option 3_12 & & & & & \\
\hline Option 3_2 & Pt black & 0.87 & $\mathrm{IrO}_{2} / \mathrm{Pt}$ & $0.73 / 0.73$ & Segmented (stripes) \\
\hline
\end{tabular}


All MEAs were tested in a single cell with Sigracet SGL 35 DC backings and a parallel serpentine flow field. Hydrogen and oxygen were fed as reactant gases because of the reversibility of the system. Normally the cell temperature in fuel cell mode was between 80 and $85^{\circ} \mathrm{C}$ and in electrolysis mode around $95^{\circ} \mathrm{C}$. The gas flow reached $250 \mathrm{sccm}$ at the anode and $200 \mathrm{sccm}$ at the cathode. Running in fuel cell mode both gases were fully humidified.

For the EIS measurements an IM6/PP240 (Zahner GmbH) and for polarization curve determination an electronic load EL 1000 (Zentro GmbH) were used.

All MEAs (except Option 3_11) are tested in the same way: Starting with the electrolysis mode (EC), polarization curves (UI) and electrochemical impedance spectroscopy (EIS) are recorded in a timeframe of about 6 to 8 hours. Afterwards the cell is switched to the fuel cell mode (FC) and the different analyses were repeated.

\section{Results and Discussion}

Before comparing the different configurations some variations in cell design for Option 2 and Option 3 and their behavior are mentioned in the following. Since Option 1 is chosen as the reference design no further investigations were carried out and it is only used for comparison.

\section{Option 2}

When investigating multilayer MEAs the order of the electrodes is of high interest. In Figure 2 one of the possible configurations is shown. Here the platinum electrode for the fuel cell mode is next to the membrane and $\mathrm{IrO}_{2}$ on top of that. This configuration is named Option 2_1. In the other configurations, called Option 2_2x, the Pt-layer is located at the outer side and $\mathrm{IrO}_{2}$ next to the membrane. By using SEM and EDX a closer look at the electrode is possible and the materials were identified (Figure 2).
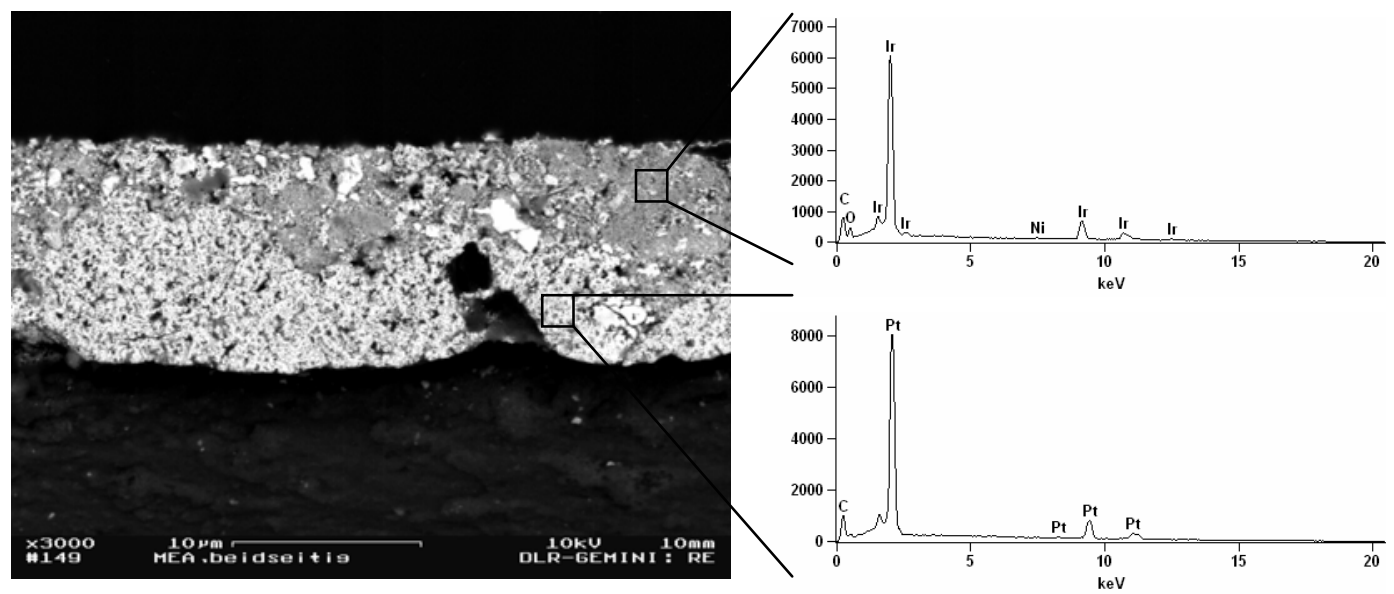

Figure 2. Electrode of an option 2 MEA with the platinum layer inside (SEM and EDX)

The polarization curves of all Option 2 MEAs are shown in Figure 3. It has to be mentioned that Option 2_1 and Option 2_22 were sprayed at one day while the other three MEAs were manufactured later. A comparison of Option 2_1 and Option 2_22 shows the difference according to the sequence of the catalyst layers. The highest performance is always obtained by the MEA with the catalyst needed for the specific 
mode next to the membrane (Option 2_1 with Pt inside best FC, Option 2_22 with $\mathrm{IrO}_{2}$ inside best EC). This behavior shows the dependence of the performance on the ionic conductivity of the electrode - membrane interface. If the reaction occurs in the outer layer, the distance to the ionomer is rather extended and the cell resistance increases significantly. Further the supply of the inner electrode is reduced because of the microporous structure in the outer layer. This effect is similar to the diffusion limitation at high current density in a generic fuel cell.

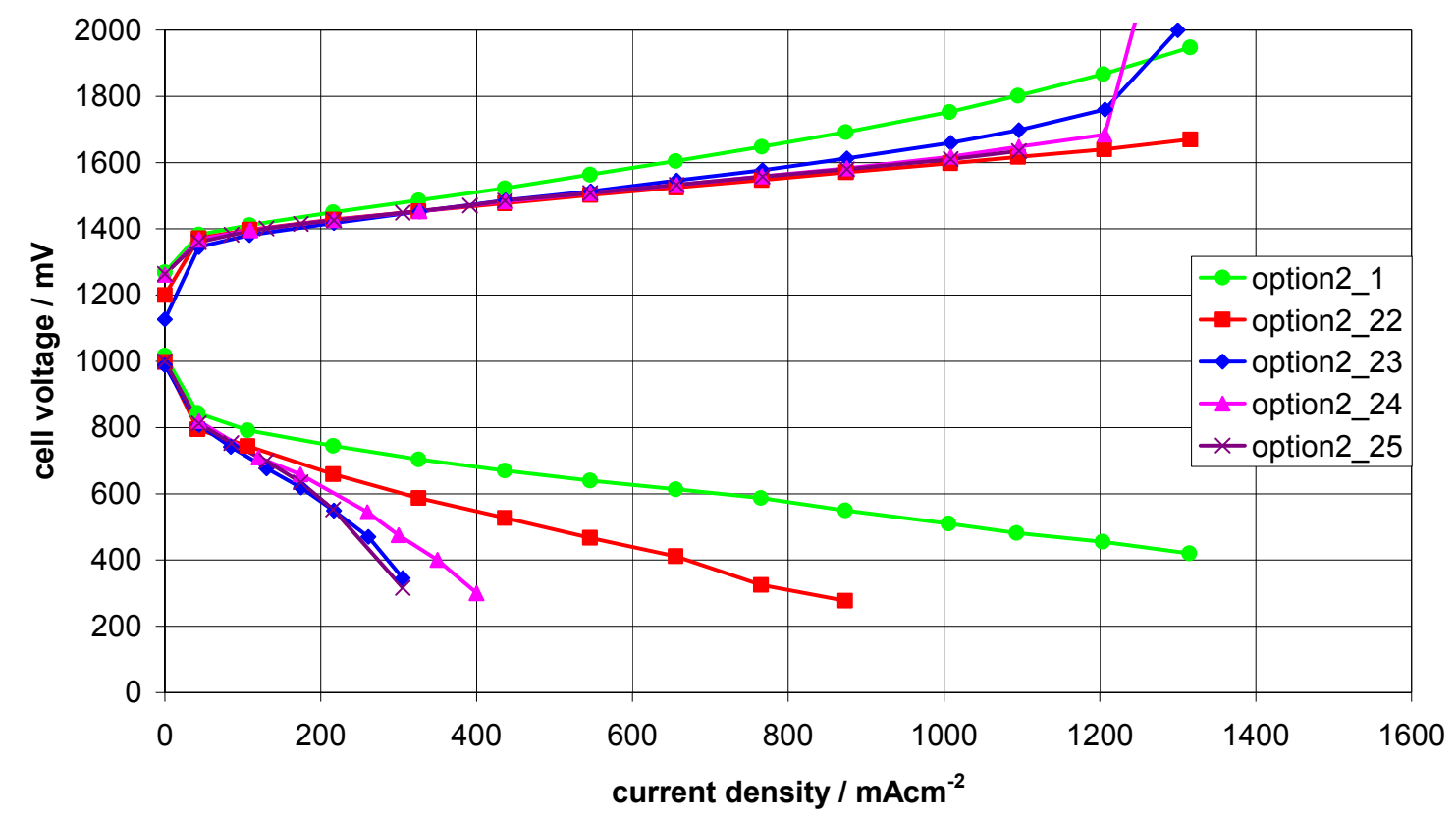

Figure 3. Polarization curves of Option 2 MEAs

In a second study the influence of the inner electrode and therefore the distance of the outer catalytic layer to the membrane is investigated. Three MEAs with increasing loadings of the inner $\mathrm{IrO}_{2}$ layer were tested (Option 2_23, Option 2_24 and Option 2_25). As expected the power in electrolysis mode decreases with increasing catalyst loading. Operation in fuel cell mode shows a rather complex behavior. The performance, also in comparison to the other Option 2 cells, shows a very strong dependence on ionic and electrical conductivity of the layer thickness. In a case of very low loading (Option 2_23) the ionic conductivity of Nafion ionomer is low and therefore the cell resistance increases. With a thick layer the bulk resistance of the inner electrode increases and the cell voltage decreases.

\section{Option 3}

Using a segmented electrode approach two basic geometries were selected. The two investigated configurations are shown in Figure 4. In Option 3_1 the catalysts were applied in squares onto the membrane and in Option 3_2 the different catalyst were applied as stripes. The results of the Option 3 MEAs are presented in Figure 5. 


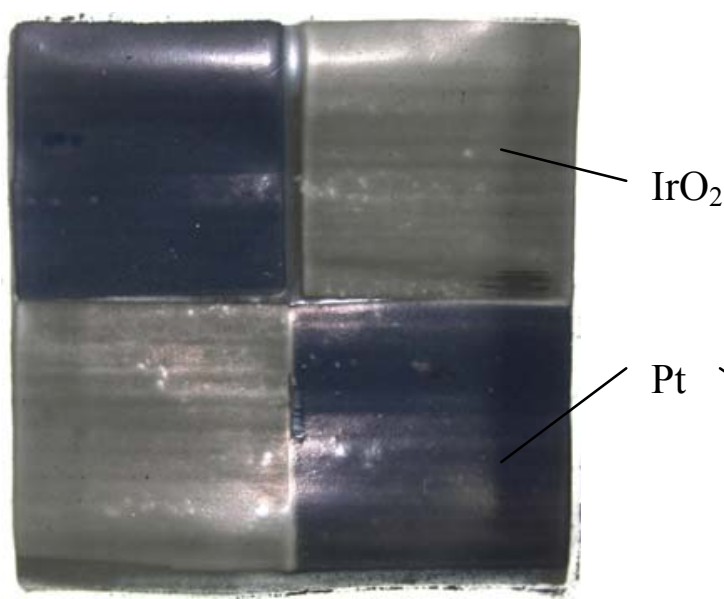

Figure 4. Segmented electrodes configurations investigated: left handed squared configuration (Option 3_1), right handed striped configuration (Option 3_2)

The Option 3_1 cells with the squared segments showed a strong sensitivity to the operation mode. The MEA Option 3_11 was used in fuel cell mode first and afterwards as electrolysis cell. The order for Option 3_12 MEA was inverted. The polarization curves of both can be seen in Figure 5. The reason for their behavior is probably a changing of the backing (e.g. surface or structure) by the different conditions of each mode, similar to the start-up of ordinary cells. The detailed processes between electrode and backing are not clarified yet.

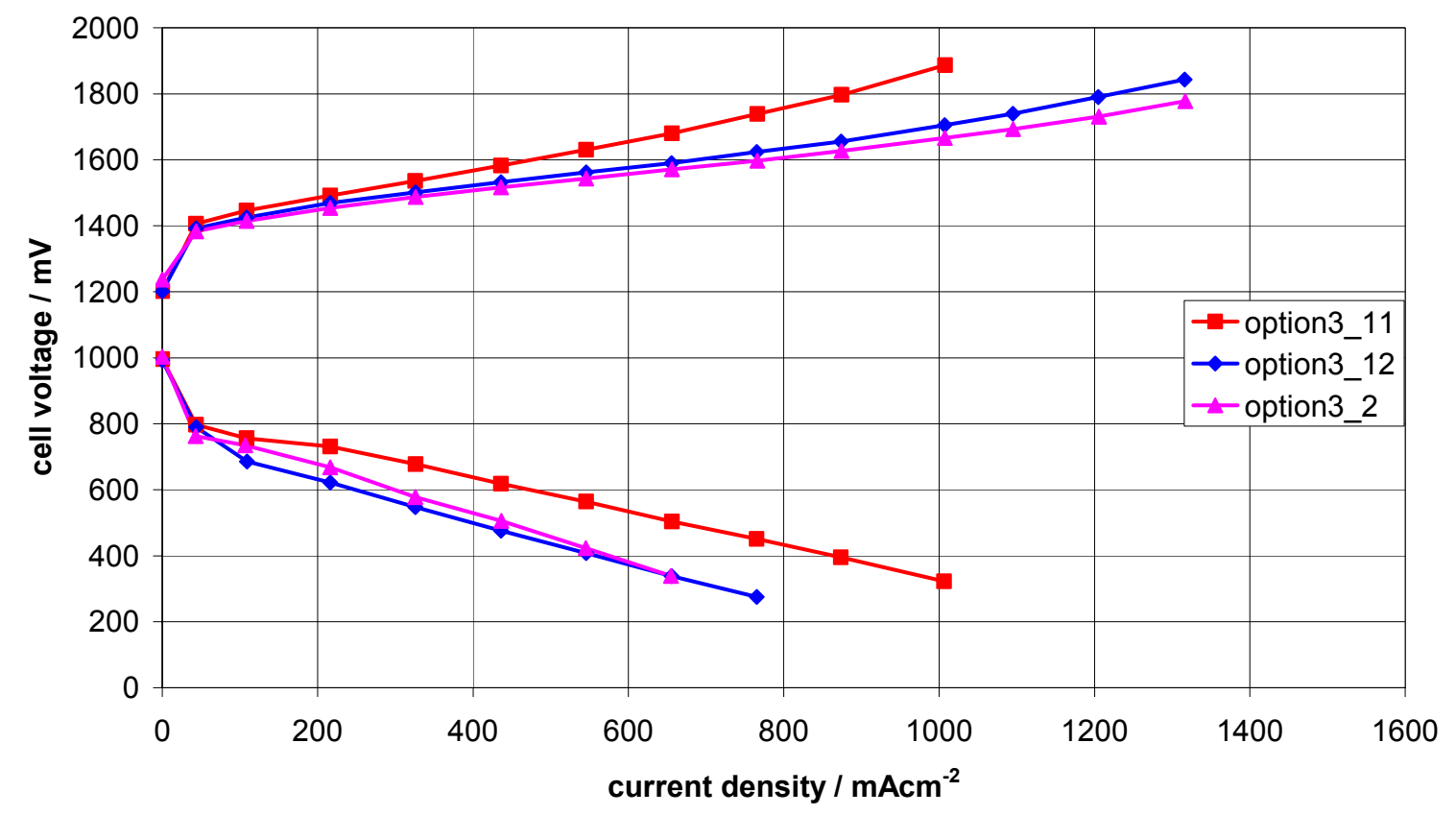

Figure 5. Polarization curves of Option 3 MEA's

The Option 3_2 MEA did not show any dependence on the working mode sequence. Also it showed better overall performances and increased stability. By comparing Option $3 \_2$ with generic electrolysis and fuel cell MEAs the activity of the catalysts in different working modes can be predicted. In electrolysis mode the performance of the segmented electrodes is slightly better than a generic electrolysis electrode with the same amount of $\mathrm{IrO}_{2}$. This shows that Pt does not reduce the overall activity for oxygen evolution, but 
rather increases it. However, in fuel cell mode the U(i) curve is lower compared to a Pt fuel cell electrode. Therefore the $\mathrm{IrO}_{2}$ hampers the fuel cell reaction to some extend. Further investigations are necessary to determine the nature of this influence (electronic conductivity versus reactivity).

Figure 6 shows the best performing polarization curves of all three options. Since the fuel cell mode was selected as the more important operation mode for a user, the best fuel cell curves are plotted. Option 1, the state-of-the-art cell design, is chosen to be the reference system and therefore the other options are compared to this configuration.

The Option 3 MEA shows the lowest performance in both modes. The reduced active area and a non-segmented backing are the reasons for this result. An optimization of the cell, especially the use of segmented backings, could improve the performance of this type.

Looking at the other MEAs, the Option 2 MEA has advantages in fuel cell mode while the Option 1 MEA shows better performance in electrolysis especially at high current densities. However, normally a fuel cell runs at about $700 \mathrm{mV}$ and at this voltage the power of both cells is identical. Because the electrolysis of the first option is superior to the second option, at this development stage the mixed catalysts structure is preferable for a technical application. An improvement of the performance in multilayer electrodes is possible by applying a higher content of Nafion in the inner and maybe a pore former (to reduce the diffusion limitation) in the outer electrode.

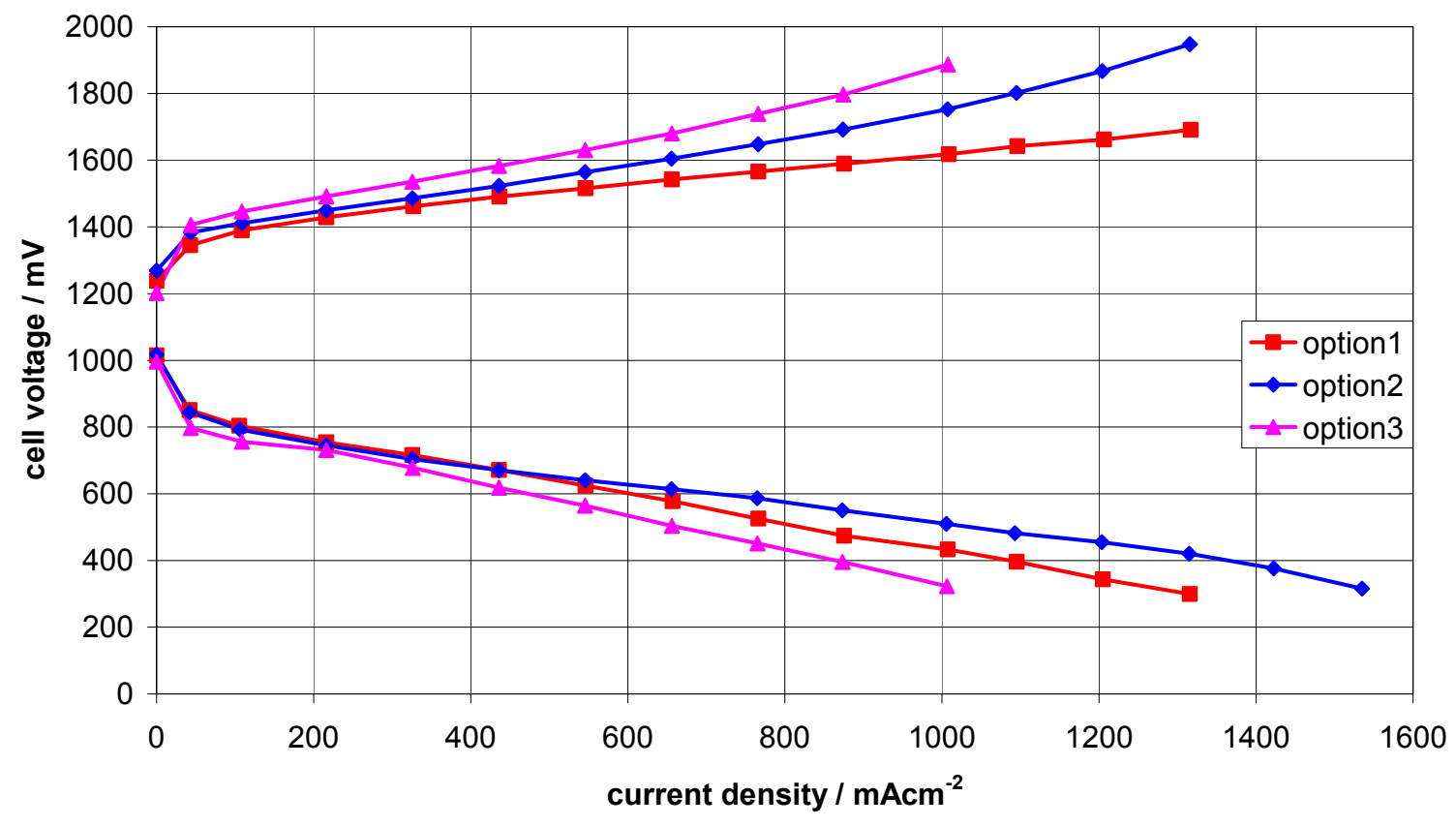

Figure 6. Polarization curves of best performing MEAs

In addition to the polarization curves some electrochemical impedance spectra have been recorded and are plotted in Figure 7 as Bode diagram. Since the shape of the spectra is completely different for electrolysis and fuel cell mode two spectra are displayed. First, the cell impedance (low frequency end of the spectra) and also the ohmic resistance (high frequency end of the spectra) are much smaller in electrolysis than in fuel cell mode. Mainly the higher water content and therefore the increased ionic conductivity of the membrane are considered to be responsible for that. 

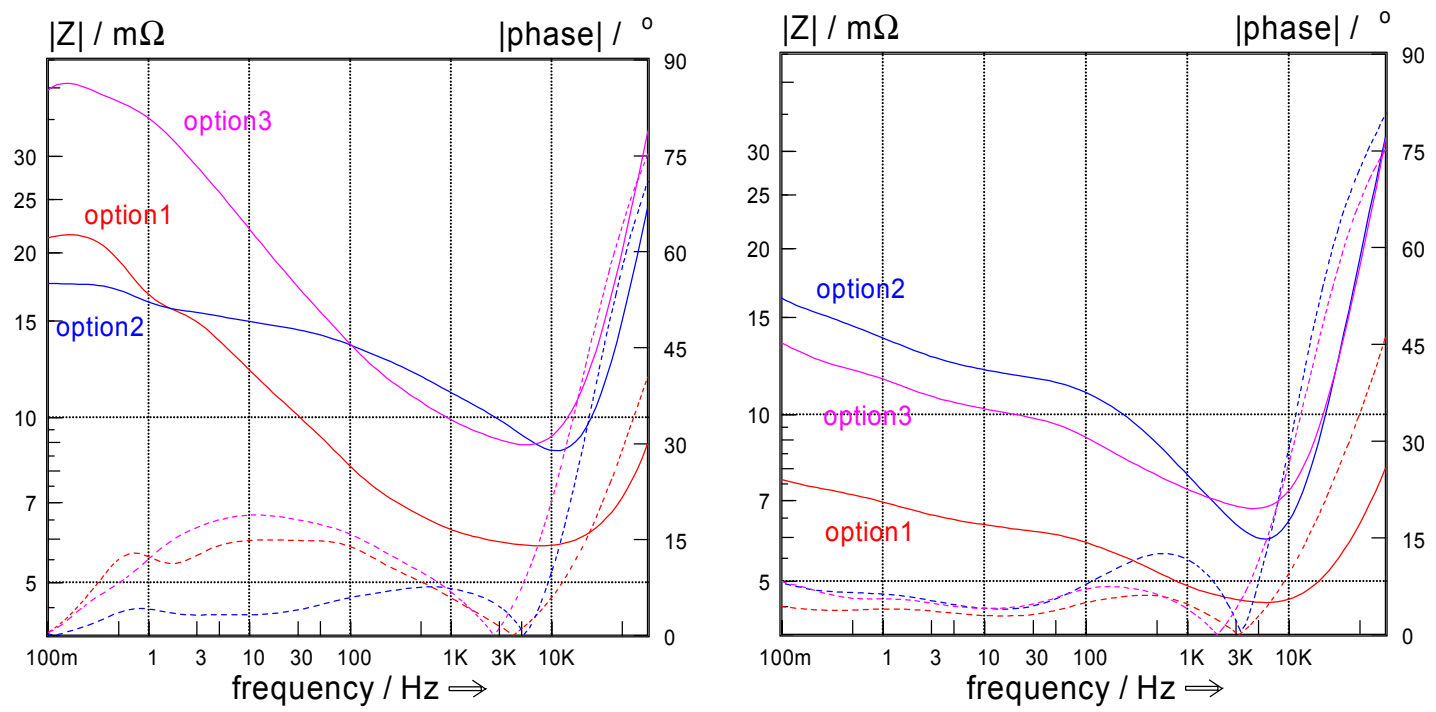

Figure 7. Impedance spectra of best performing MEAs. Left: fuel cell mode; Right: electrolysis mode

Comparing the different curves the Option 3 MEA shows the highest cell impedance in fuel cell mode, which is in accordance with the performance in the polarization curves. The reason is the reduction of the actual active area by two. Normalizing the impedance might show a different plot. The Option 2 MEA has the smallest cell impedance but a higher ohmic resistance. This difference at high frequencies to Option 1 is attributed to the much thicker electrode and the reduced electrical conductivity of the pure $\mathrm{IrO}_{2}$ layer.

In electrolysis mode the Option 1 MEA has the smallest impedance and therefore the best performance. Unexpectedly, the Option 2 MEA shows the highest impedance. An explanation may be the diffusion limitation by the multilayer configuration reduces the water transport to the electrode and leads to an increased resistance.

\section{Conclusions}

In this work different oxygen electrode configuration for the operation in a unitized reversible fuel cell are compared. Different possibilities for bifunctional electrodes based on the primary catalysts are compared, namely a mixture of catalysts, layered structures with the two different catalyst layers, and segmented areas with single catalysts. It was shown that the mixture of both catalysts $\left(\mathrm{Pt}\right.$ and $\left.\mathrm{IrO}_{2}\right)$ performs best for the present stage of electrode development. Also multilayer electrodes yielded good results with a good potential of optimization.

For all MEAs only a short-term stability was achieved. One challenge for further development is the increase of lifetime. Primarily the optimization of the coal-based backing and using different materials for them should be considered.

\section{References}

1. E. Rasten, G. Hagen, R. Tunold, Electrochimica Acta, 48, p. 3945 - 3952 (2003).

2. H. Wendt, Electrochemical hydrogen technologies electrochemical production and combustion of hydrogen, Elsevier, Amsterdam, (1990).

3. T. Ioroi, N. Kitazawa, K. Yasuda, Y. Yamamoto, H. Takenaka, Journal of The Electrochemical Society, 147(6), p. 2018 - 2022 (2000). 
4. H. Liu, B. Yi, M. Hou, J. Wu, Z. Hou, H. Zhang, Electrochemical and Solid-State Letters, 7(3), p. A56 - A59 (2004).

5. E. Gülzow, M. Schulze, N. Wagner, T. Kaz, R. Reissner, G. Steinhilber, A. Schneider, Journal of Power Sources, 86, p. 352 - 362 (2000). 